\title{
Wrigley Prophylaxe Preis 2017
}

Wissenschaftler, Ärzte und andere Akademiker können sich bis zum 1 . März nächsten Jahres um den mit $10000 €$ dotierten Wrigley Prophylaxe Preis 2017 bewerben. Dazu eingeladen sind alle, die sich für die Verbesserung der Zahn- und Mundgesundheit der Bevölkerung in Wissenschaft, Praxis und öffentlichem Gesundheitswesen einsetzen. Neben dem Wrigley Prophylaxe Preis wird dieses Jahr be-

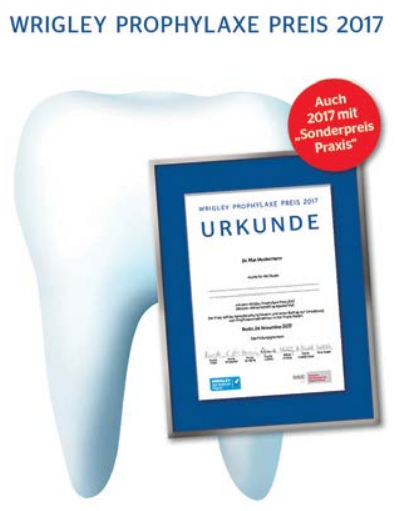

Unter der Schirmherrschaft der $\mathbf{D Q Z}$

reits zum 4. Mal der mit $2000 €$ dotierte Sonderpreis „Niedergelassene Praxis und gesellschaftliches Engagement" ausgeschrieben. Die Gesundheitsinitiative Wrigley Oral Healthcare Program (WOHP) zeichnet 2017 zusammen mit der Deutschen Gesellschaft für Zahnerhaltung (DGZ) bereits zum 23. Mal die besten Arbeiten und Projekte zur Prävention oraler Erkrankungen aus. Ziel des Preises ist die Verbesserung der Zahn- und Mundgesundheit aller Bevölkerungsgruppen.

Informationsflyer und Bewerbungsformulare mit Teilnahmebedingungen - auch zum „Sonderpreis Praxis“ - sind unter www.wrigley-dental.de abrufbar oder bei kommed Dr. Bethcke, Fax: 089/330364 03, info@kommed-bethcke.de erhältlich. 